chemotherapie-Arm möglich. Somit hat die postoperative Radiochemotherapie im perioperativen Konzept keinen Stellenwert. Wichtige Subgruppenanalysen (z. B. intestinale Tumoren, $\mathrm{N}+$ oder R1) werden nachfolgend präsentiert. $\mathrm{Zu}$ künftige Studienformate richten sich auf die präoperative Behandlungsphase aus, unter anderem die EORTC-TOPGEAR-Studie.

$\mathrm{Zu}$ den fortgeschrittenen Stadien des Magenkarzinoms wurden keine reifen Phase-III-Daten präsentiert. Vielversprechend, zumindest für einen Teil der
Patienten, sind Immuntherapien mit den bekannten Checkpointinhibitoren (anti-PD-L1 oder anti-PD-1, auch kombiniert mit anti-CTLA-4).

Als Neuigkeit wurde ein tight-junction-assoziiertes Protein (Claudin 18.2) präsentiert, welches sich durch den chimären monoklonalen Antikörper IMAB362 therapeutisch adressieren lässt [Al-Batran S et al. ASCO. 2016;Abstr LBA4001]. In einer randomisierten Phase-II-Studie zeigte die Kombination aus Chemotherapie plus IMAB362 im Vergleich zur Chemotherapie alleine eine Verbesserung des PFS (median 7,9 vs. 4,8 Monate; HR 0,47; $\mathrm{p}=0,001$ ) und OS (median 13,2 vs. 8,4 Monate; HR $0,51 ; \mathrm{p}=0,0001)$ bei Patienten mit CLD18.2-positivem Magenkarzinom im Stadium IV.

Prof. Dr. med.
Florian Lordick
Universitäres Krebszen-
trum Leipzig (UCCL), Uni-
versitätsklinikum Leipzig
Florian.Lordick@medizin.
uni-leipzig.de

\title{
Fortschritte in der Therapie urogenitaler Tumoren
}

Im Bereich der urogenitalen Tumoren spielte die Immuntherapie eine zentrale Rolle. Im Fokus standen dabei insbesondere die Ergebnisse zur PD-L1-/PD1-Inhibition in der Behandlung fortgeschrittener Urothelkarzinome.

So wurden die Daten beider Kohorten der IMvigor210-Studie mit Atezolizumab vorgestellt. Ein Update der Kohorte 2 zur Therapie platinvorbehandelter Patienten zeigte eine Ansprechrate (RR) von $16 \%$ bei allen Patienten - mit einer RR von $28 \%$ bei einer PD-L1-Expression $\geq 5 \%$. In dieser Subgruppe konnte ein komplettes Ansprechen (CR) bei $15 \%$ der Patienten erzielt werden, welches zum Auswertungszeitpunkt bei $86 \%$ fortbestand [Dreicer R et al. ASCO. 2016; Abstr 4515]. Erstmalig wurden zudem Ergebnisse zur Erstlinientherapie mit Atezolizumab bei Cisplatin-ungeeigneten Patienten (Kohorte 1) präsentiert. Mit $70 \%$ war eine eingeschränkte Nierenfunktion Hauptkriterium für den Einschluss in diese Gruppe. Die ORR betrug $24 \%$, mit $7 \%$ CR und $17 \%$ partiellen Remissionen (PR). Hier hatte der PD-L1-Status interessanterweise keinen signifikanten Einfluss auf die ORR, die Zahl der CR oder das OS [Balar AV et al. ASCO. 2016;Abstr LBA4500].

In der Studie CheckMate 032 erzielte der PD-1-Inhibitor Nivolumab eine ORR von $24,4 \%$, mit $6,4 \%$ CR in einer stark vorbehandelten Patientengruppe (> $65 \%$ mit $\geq 2$ Vortherapien). Auch hier hatte der PD-L1-Status keinen Einfluss auf das Behandlungsergebnis [Sharma $\mathrm{P}$ et al.
ASCO. 2016;Abstr 4501]. Hingegen war das PD-L1-Expressionsmuster für die Wirksamkeit des gegen PD-L1 gerichteten Antikörpers Durvalumab in der $\mathrm{Pa}$ tientengruppe mit Urothelkarzinom der 1108 -Studie ausschlaggebend. So zeigten $46 \%$ der Patienten mit einer PD-L1-Espression > 25\% eine ORR, während dies bei keinem der PD-L1 negativen Patienten der Fall war [Massard C et al. ASCO. 2016; Abstr 4502].

Für den Behandlungsalltag unmittelbar relevante Ergebnisse wurden für das Prostatakarzinom präsentiert. Patienten mit metastasiertem Prostatakarzinom, die in der CHAARTED-Studie frühzeitig eine Kombination aus Docetaxel und Androgendeprivationstherapie (ADT) erhalten hatten, wiesen nicht nur ein besseres OS auf als Patienten mit alleiniger ADT, sondern nach einem Jahr auch eine bessere Lebensqualität, obwohl diese zunächst im Rahmen der Chemotherapie abgenommen hatte. [Patrick-Miller LJ et al. ASCO. 2016; Abstr 5004]. Interessanterweise zeigte die adjuvante Chemotherapie mit Docetaxel (ohne ADT oder kontinuierliche Steroidgabe) bei Hochrisikopatienten keinen Vorteil im Vergleich zu einer alleinigen Überwachung [Ahlgren G et al. ASCO. 2016;Abstr 5001].

In der PROSELICA-Studie konnte bei vorbehandelten Patienten mit kastrationsresistentem Prostatakarzinom (CRPC) nachgewiesen werden, dass die niedrigere Cabazitaxeldosierung von 20 $\mathrm{mg} / \mathrm{m}^{2}$ der Standarddosierung bezüglich des OS nicht unterlegen ist [De Bono JS et al. ASCO. 2016;Abstr 5008]. In der FIRSTANA-Studie erzielten Docetaxel und Cabazitaxel (in den Dosierungen $20 \mathrm{mg} / \mathrm{m}^{2}$ und $25 \mathrm{mg} / \mathrm{m}^{2}$ ) in der Erstlinientherapie bei Patienten mit CRPC ein vergleichbares OS und PFS. Unterschiede bestanden dagegen im Nebenwirkungsprofil mit einer Rate febriler Neutropenien von $12 \%$ unter $25 \mathrm{mg} / \mathrm{m}^{2} \mathrm{Ca}$ bazitaxel, 8,3\% unter Docetaxel, aber nur 2,4\% unter $20 \mathrm{mg} / \mathrm{m}^{2}$ Cabazitaxel. Hämaturien kamen in beiden Cabazitaxel-Behandlungsgruppen deutlich häufiger vor. Hingegen traten Ödeme, Polyneuropathien und Stomatitis vermehrt bei Docetaxel-behandelten Patienten auf [Sartor AO et al. ASCO. 2016;Abstr 5006].

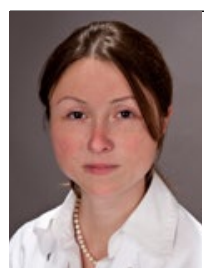

PD Dr. med. Gunhild von Amsberg Klinik für Onkologie, Hämatologie und Knochenmarkstransplantation mit Sektion Pneumologie Universitätsklinikum Hamburg-Eppendorf g.von-amsberg@uke.de

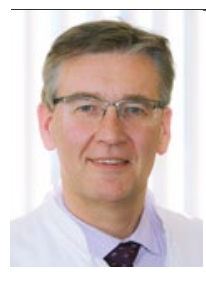

Prof. Dr. med. Carsten Bokemeyer Klinik für Onkologie, Hämatologie und Stammzelltransplantation mit Sektion Pneumologie, Universitätsklinikum Hamburg-Eppendorf cbokemeyer@uke.de 